\title{
Rates of oropharyngeal cancer continue to rise steeply amongst Australian men: continued emphasis on HPV vaccination, tobacco and alcohol control
}

Ka Ming Fan

Griffith University Queensland

Samuel Sprague

Griffith University Queensland

Ping Zhang

Griffith University Queensland

Anura Ariyawardana

Queensland Health

Newell W. Johnson ( $\nabla$ n.johnson@griffith.edu.au )

Griffith University Queensland

\section{Research Article}

Keywords:

Posted Date: March 2nd, 2022

DOI: https://doi.org/10.21203/rs.3.rs-1403605/v1

License: (c) (1) This work is licensed under a Creative Commons Attribution 4.0 International License.

Read Full License 


\section{Abstract \\ Objective}

To analyse trends in incidence of oropharyngeal cancers (OPC) across Australia from 1982 to 2017 with implications for prevention.

\section{Methods}

Data were obtained from the Australian Cancer Database (ACD) compiled at the Australian Institute of Health and Welfare (AIHW). Incidence rates of OPC were expressed per 100,000 population, agestandardized in accordance with WHO standard population. Joinpoint analyses are presented.

\section{Results}

A striking increase of age-standardised incidence rate (ASIR) of OPC by over 1.5 times from 1.86/100,000 in 1982 to $3.23 / 100,000$ in 2017 was observed in men: the most significant rise was between 2007 and 2017 with an annual percentage change (APC) of $+5.24 \%(p<0.001)$. Slow but gradual growth of ASIR from $0.38 / 100,000$ in 1982 to $0.71 / 100,000$ in 2017 was observed among women with a statistically significant APC of $+1.02 \%(p<0.001)$. Statistically significant bimodal increasing trends of APC were also observed in total ASIR of OPC. Highest number of incident cases were found in patients aged 55 to 69 years old, accounting for $50.8 \%$ on average of all ages during each 7-year time period. Most common subsites affected were base of tongue (BOT) and "oropharynx" from 1982 to 2017.

\section{Conclusion}

OPC is rising rapidly across Australia, particularly in men. Whilst the national proportion of cases driven by HPV is not known, it is evident that vaccination is yet to have an impact. A substantial proportion of cases are, presumably, still driven by traditional alcohol and tobacco habits, mandating continued efforts at control of these risk factors.

\section{Introduction}

Rates of oropharyngeal cancers (OPC) have been rising in Australia for several decades (1), as in other developed countries (2). It is evident that specific human papillomavirus (HPV) infections are a major risk factor for HNC, particularly for OPC, mainly genotypes 16 and 18. With over $200 \mathrm{HPV}$ genotypes identified, $13 \mathrm{HPVs}$ are classified as Group I carcinogens by the International Agency for Research on Cancer (IARC), namely HPV 16, 18, 31, 33, 35, 39, 45, 52, 56, 58, 59, 61 and 66 (3). Two studies in Australia reported that $47.7-57.3 \%$ of OPC cases were HPV positive, with HPV type 16 being the most prevalent $(91-95 \%)(4,5)$. 
Notably, in Australia, girls and boys aged 12-13 years have been eligible to receive vaccination against HPV since 2007 and 2013, respectively, in order to protect them from cervical cancer, genital warts, and other HPV-related diseases including OPC (6). With Australia being one of the 27 countries globally which have extended HPV vaccination to males, two HPV vaccines are currently available under the National Immunisation Program (NIP) for both sexes (7). Cervarix is a bivalent HPV vaccine protecting against oncogenic types 16 and 18, while the nonavalent HPV vaccine, Gardasil 9, targets 7 additional HPV types $(6,11,31,33,45,52$, and 58) (6). Gardasil, a former quadrivalent HPV vaccine (HPV 6, 11, 16, and 18), was introduced in 2007 but replaced by Gardasil 9 in 2018 (8). By 2017, 75.9\% and 80.2\% of males and females aged 15 respectively had received their third dose of Gardasil, vaccination rates having rising annually since 2007 (9). As a result, the prevalence of oncogenic HPVs in female genital samples fell from $29-7 \%$ in cross-sectional studies conducted before (2005-2007) and after (2010-2012) the introduction of HPV vaccines (10): national changes in carriage in the upper aerodigestive tract are, however, yet to be demonstrated.

Nonetheless, tobacco use and heavy alcohol consumption remain well-established major risk factors for OPC, as with other types of head and neck cancers (HNC), often in communities of low socio-economic status and often in subjects with diets lacking in antioxidant vitamins and minerals (11). A pooled analysis from the International Head and Neck Cancer Epidemiology Consortium concluded that $33 \%$ of HNC were caused by tobacco use alone, compared to only $4 \%$ when only alcohol was involved: $35 \%$ of HNC cases were attributed to a combination of tobacco and alcohol (12).

\section{Objectives}

We previously described the incidence of oral cavity and of oropharyngeal cancer across Australia between 1982 and 2008 (1). The objectives of the present study were to update incidence trends of OPC from 1982-2017, to describe differences by sex and age, and to differentiate trends by subsites within the oropharyngeal region. We also speculate the likely influences of HPV vaccination on incidence trends of OPC during the study period and in the future, separately for males and females.

\section{Methods}

Data on oropharyngeal cancers from 1982 to 2017 were obtained from the Australian Cancer Database (ACD) compiled at the Australian Institute of Health and Welfare (AlHW) with approvals from all Australian cancer registries, specifically New South Wales, Northern Territory, Queensland, South Australia, Tasmania, Victoria, and Western Australia.

The raw data include sex and year of diagnosis, from 1982 to 2017 . Age at diagnosis was expressed in 19 distinct five-year age groups: $0-4,5-9,10-14,15-19,20-24,25-29,30-34,35-39,40-44,45-49$, $50-54,55-59,60-64,65-69,70-74,75-79,80-84,85-89$, and 90 or above. Data were available according to anatomical site codes for malignant neoplasms of lip, oral cavity and pharynx (codes C00.0 to C14.9) and 4-digit histology codes were provided according to the World Health Organization 
International Statistical Classification of Diseases and Related Health Problems 10th Revision (ICD-10), and the International Classification of Diseases for Oncology, Third Edition, First and Second Revisions (ICD-0-3.1 and 3.2), respectively.

Subsites of OPC were base of tongue (BOT) (C01), lingual tonsil (C02.4), soft palate (C05.1), uvula (C05.2), (palatine) tonsil (C09), oropharynx (C10), posterior wall of nasopharynx (C11.1), and Waldeyer's ring (C14.2).

Incidence rates (IR) of OPC were calculated and expressed per 100,000 population. Age-standardized incidence rates (ASIR) were calculated in accordance with WHO standard population and expressed per 100,000 population. Statistical software R version 4.1.2 was used to manipulate raw data and generate figures. Estimated resident population (ERP) was obtained from Australian Demographic Statistics version 3101.0, released by the Australian Bureau of Statistics. Joinpoint trend analyses were performed with the software released by US National Cancer Institute, Division of Cancer Control and Population Sciences, available at https://surveillance.cancer.gov/joinpoint/.

\section{Results}

A total of 13,185 cases of OPC were reported during the period from 1982 to 2017 . Of these, 10,285 $(78.0 \%)$ were in males and 2,900 (22.0\%) in females, a male-to-female ratio of 3.55:1. Histologically, $90.7 \%$ of all incident cases were classified and collectively grouped under squamous cell neoplasms: 8070-squamous cell carcinoma (SCC), not otherwise specified (NOS) (71.2\%), 8071-SCC, keratinizing, NOS (11.1\%), 8072-SCC, large cell, non-keratinizing, NOS (4.8\%), and 8083-basaloid squamous cell carcinoma (3.6\%).

Figure 1 shows the result of the joinpoint analysis of ASIR from 1982 to 2017 for males. A striking 1.5 times increase in ASIR was found from 1.86/100,000 in 1982 to 3.23/100,000 in 2017: the most significant rise was between 2007 and 2017 with an annual percentage change (APC) of $+5.24 \%(p<$ 0.001 ) at a significance level of $p<0.05$. As shown in Fig. 2, a comparatively less but gradual growth of ASIR from $0.38 / 100,000$ in 1982 to $0.71 / 100,000$ in 2017 was observed among women, with a statistically significant APC of $+1.02 \%(p<0.001)$. Figure 3 demonstrates the increase in ASIR from $1.10 / 100,000$ in 1982 to $1.94 / 100,00$ in 2017 for combined males and females. Two significant increasing trends of APC were identified: $+2.21 \%$ from 1982 to $1992(p=0.003)$, and $+4.51 \%$ from 2006 to $2017(p<0.001)$.

Figure 4 shows the number of incident cases in each age group in 7-year periods from 1983 to 2017. Highest number of incident cases were found in patients 55 to 69 years old (age groups 12 to 14), accounting for $50.8 \%$ on average (range $45.0-54.4 \%$ ) of all ages during each 7-year time period, while the mean of proportion of cases in patients aged 40 to 59 (age groups 9 to 12) was 40.0\% (range 35.642.9\%). These proportions, however, fluctuated slightly during the study period. In contrast, the proportion of cases in patients aged 80 to 85 + years (age groups 18 to 19) increased in each time period from $1.5 \%$ in $1983-1989$ to $2.6 \%$ in $2011-2017$. 
*Age group $1=0-4$ years, $2=5-9$ years, $3=10-14$ years, $4=15-19$ years, $5=20-24$ years, $6=25-29$ years, $7=30-34$ years, $8=35-39$ years, $9=40-44$ years, $10=45-49$ years, $11=50-54$ years, $12=55-$ 59 years, $13=60-64$ years, $14=65-69$ years, $15=70-74$ years, $16=75-79$ years, $17=80-84$ years, 18 $=85-89$ years, $19=90$ years or above.

Figure 5 demonstrates the incidence of OPC in eight anatomical subsites. BOT (C01) was overall the most affected subsite, showing a three-time increase in IR from 0.58/100,000 in 1994 to $1.75 / 100,000$ in 2017. Oropharynx (C10), as the second most affected subsites after 1990, also showed an over 2.5 times rise in IR from $0.28 / 100,000$ in 2006 to $0.78 / 100,000$ 2017. Relatively low yet stable IR were seen in lingual tonsil, uvula, soft palate, palatine tonsil, and pharyngeal tonsil from 1982 to 2017 . Only one case of OPC was classified as arising in Waldeyer's ring, but this anatomical designation overlapped with many of the above site codes.

*Only one case of carcinoma of the Waldeyer's ring was diagnosed in 2003 , thus its IR was negligible

\section{Discussion}

The current study demonstrates a continuation of the trends we reported previously (1). There is a further acceleration of OPC cases from 2008 to 2017, especially in males. This, in spite of falling tobacco and alcohol consumption in Australia. As reported by AlHW, the proportion of daily smokers aged 14 or above in Australia decreased from $24 \%$ in 1991 to $11 \%$ in 2019, and the percentage of never-smokers rose from 49\% in 1991 to $63 \%$ in 2019 (13). According to Australian Guidelines to Reduce Health Risks from Drinking Alcohol, consuming more than 2 standard drinks per day can lead to a lifetime risk of alcoholrelated diseases and hence of OPC (14). This type of alcohol use decreased by about $4 \%$ of the population from 2001 (21\%) to 2019 (16.8\%), but nearly a quarter of Australian males (24\%) still exceeded this lifetime risk behaviour, 2.5 times higher than that in females $(9.4 \%)$ in 2020. Australians aged 15 and over consumed 9.5 litres of alcohol per capita in 2021, more than the 8.7 litres per capita reported for 2020 (13). Despite the overall reduction in these established risk factors, it is likely that tobacco and alcohol remain significant causes of OPC, especially in older Australians. When case series indicate that about $50 \%$ of recent OPC cases are related to tobacco and excessive alcohol use (5), it is clear that continued efforts on tobacco and alcohol control are needed, even though this proportion is likely to decrease in the future.

No national data on the proportion of cases associated with HPV are available. Nevertheless, it is reasonable to propose that a significant proportion of the rise in incidence of OPC is HPV driven, especially amongst younger cohorts. BOT cancer was the most common OPC during the study period in Australians. HPVs are most commonly detected at this subsite and are likely to be causally related to the rise in cancers here (15). Tonsillar cancers, which have a high association with HPV infection (15), constituted a relatively low IR compared to BOT and "oropharynx" in these Australian data. This finding is in contrast to data from a study of all HNC in Australia, in which "tonsillar" cancers had a higher ASIR than BOT cancers from 1982 to 2005: these authors considered only what they regarded as potentially 
HPV-associated OPC and applied different subsite codes (16). Further studies are needed to clarify differences by subsite.

The age distribution of OPC resembled a normal distribution with peaks at 55 to 69 years from 1982 to 2017. This relatively late onset of OPC is likely due to the time required for cumulative and persistent damage by HPV, tobacco, or alcohol to the oropharynx (11). 40\% of our OPC cases were aged 40 to 59 years, an age bracket with the highest incidence of HPV-driven OPC according to epidemiological studies in the USA (17).

Early age at oral sexual debut, past experience with oral sex (OS), and high number of oral sexual partners (OSP) are major risk factors of persistent oral and oropharyngeal HPV infection (18). This in turn increases the risk of developing HPV-related OPC (19). Studies have found that younger Australians tend to initiate OS at an earlier age than heretofore, and this is becoming more prevalent (20). Australians born between 1981 and 1986 had their first OS almost 7 to 10 years earlier than those born between 1941 and 1950 (20). Among $85.8 \%$ of males and $80.1 \%$ of females who reported ever having OS between 2012 and 2013 , about $55 \%$ had first performed this activity at 17 years or younger (21). Individuals with previous OS experience and initiation of OS before 18 years of age are 4.4 and 1.8 times more likely to develop HPV-driven OPC than those who had never performed OS or had initiated OS at 20 years or above, respectively (18). Unfortunately, no Australian data regarding number of lifetime OSP are available, which has been shown to have a strong association with HPV-positive OPC in populations in the USA (18). Males are more likely to acquire an oral HPV infection than females, and hence are at risk of HPV-related OPC because female genital mucosa has a higher HPV viral load than male genital mucosa or skin (17). We therefore hypothesize that the rise in oral sexual practices in Australia has generated the rapid increase in HPV-positive OPC since 2006, especially in young to middle aged males. More incident cases of OPC in the next few decades will be HPV-driven, at least until vaccination programmes have evolved to a state of herd immunity.

In a recent systematic review summarizing the effects of HPV vaccination on oral and oropharyngeal HPV infection, $93.2 \%$ of the participants had developed IgG antibodies against HPV 16 in their oral fluids with an average relative prevention percentage of $83.9 \%$ (22). Nevertheless, our present data reveal that the effect of HPV vaccination is yet to be seen in terms of reduced incidence of OPC. Currently, since most incident cases of OPC arise after the age of 40, the effect of HPV immunization in Australia will not even begin to be seen until this cohort reaches high-risk ages which are at least a decade hence for women and at least two decades hence for men. Even then the impact will be slow and will not be seen in large numbers for a further decade or more because it is dependent on herd immunity, which is itself dependent on teenagers having received all three vaccine doses in significant numbers. Tracking of oral and oropharyngeal carriage of HPV 16 and 18 amongst children and adolescents (aged 9-18 years) in Australia would be beneficial even though low prevalence rates would be expected. A recent study in the USA also projected that reduced rates of OPC from HPV vaccination would only begin to be seen from around 2045 when unvaccinated older individuals become the major risk group compared with thenvaccinated younger individuals (23). 
In contrast, an effect on cervical cancer can be expected sooner because of the strong antibody response in young women and the earlier onset of cervical cancer. Indeed, there are already data suggesting Australia may be well on the way to being the first country in the world to eliminate this disease (24). Falls in the incidence of severe cervical epithelial dysplasia (CIN3) and of cervical cancer are expected in the UK after the introduction of the national HPV vaccination programme. Projection modelling in the UK shows that participants who had received their HPV vaccine at $12-13$ years showed $97 \%$ and $87 \%$ relative risk reduction for CIN3 and cervical cancer, respectively, compared to unvaccinated cohorts (25).

\section{Conclusion}

OPC is a rising concern across Australia, particularly in men. Increase in incident cases and ASIR are observed in both sexes with a strong male predilection. Tobacco and alcohol control measures remain important. However, an increasing proportion of OPC will be HPV-driven in the future until herd immunity is generated by vaccine coverage. Thus, HPV vaccination, sex education, and opportunistic screening by primary care doctors and dentists, should be reinforced.

\section{Limitations}

We have not analysed data by geographical area across Australia. It has not been possible to associate cases directly with tobacco and alcohol use or with HPV infection. The national database does not have information on HPV presence, by p16 positivity or any other method, so the proportion due primarily to $\mathrm{HPV}$ is not known. Likewise, the real role of sexual transmission can only be inferred. Available data on histological type do not permit judgement as to the proportion of cases which are lymphoid carcinomas likely to be virus-associated.

\section{Abbreviations}

APC - annual percentage change

ACD - Australian Cancer Database

AlHW - Australian Institute of Health and Welfare

ASR/ASIR - age-standardised incidence rates

BOT - base of tongue

ERP - estimated resident population

HNC - head and neck cancers

HPV - Human Papillomavirus

IARC - International Agency for Research on Cancer 
ICD-10 - International Statistical Classification of Diseases and Related Health Problems 10th Revision

ICD-0-3.1 and 3.2 - International Classification of Diseases for Oncology, Third Edition, First and Second Revisions

IR - incidence rates

NIP - National Immunisation Program

NOS - not otherwise specified

OPC - oropharyngeal cancers

OS - oral sex

OSP - oral sexual partners

CIN3 - severe cervical epithelial dysplasia

US/USA - United States of America

UK - United Kingdom

\section{Declarations}

\section{Author Contributions}

Fan, K.M.: data acquisition; data processing; interpretation and writing

Sprague, S.: data processing; interpretation and writing

Zhang, P.: data processing and interpretation

Ariyawardana, A.: interpretation

Johnson, N.W.: conceptualisation; interpretation and writing

All authors have reviewed and approved the final manuscript

\section{$\underline{\text { Conflict of Interest }}$}

No potential personal conflict of interest exists for all authors. 
Data Availability Statement

The data that support the findings of this study are available from the corresponding author upon reasonable request.

\section{Ethical Approval Statement}

Ethical approvals were not required as individuals are de-identified in this study.

\section{Acknowledgments}

We are very grateful to Dr. Mark Short of AlHW for access to the database and for his advice on cancer registration procedures.

\section{References}

1. Ariyawardana A, Johnson NW. Trends of lip, oral cavity and oropharyngeal cancers in Australia 1982-2008: overall good news but with rising rates in the oropharynx. BMC Cancer. 2013;13:333.

2. Bosetti C, Carioli G, Santucci C, et al. Global trends in oral and pharyngeal cancer incidence and mortality. Int. J. Cancer. 2020;147(4):1040-9.

3. International Agency for Research on Cancer [IARC]. Human Papillomaviruses: IARC Monographs on the Evaluation of Carcinogenic Risks to Humans; 2007.

4. Hong AM, Grulich AE, Jones D, et al. Squamous cell carcinoma of the oropharynx in Australian males induced by human papillomavirus vaccine targets. Vaccine. 2010;28(19):3269-72.

5. Hong A, Jones D, Chatfield M, et al. HPV Status of Oropharyngeal Cancer by Combination HPV DNA/p16 Testing: Biological Relevance of Discordant Results. Ann. Surg. Oncol. 2013;20(S3):450-8.

6. Australian Technical Advisory Group on Immunisation [ATAGI]. Australian Immunisation Handbook: Australian Government Department of Health, Canberra; 2018.

7. López N, Garcés-Sánchez M, Panizo MB, et al. HPV knowledge and vaccine acceptance among European adolescents and their parents: a systematic literature review. Public Health Rev. 2020;41(1):10.

8. Brotherton J. Human papillomavirus vaccination update. Australian Journal for General Practitioners. 2018;47:417-21.

9. Cancer Australia. HPV vaccination uptake National Cancer Control Indicators,2019. Available from: https://ncci.canceraustralia.gov.au/prevention/hpv-vaccination-uptake/hpv-vaccination-uptake. (accessed Dec 2021) 
10. Tabrizi SNAP, Brotherton JMLB, Kaldor JMP, et al. Assessment of herd immunity and cross-protection after a human papillomavirus vaccination programme in Australia: a repeat cross-sectional study. The Lancet Infect. Dis. 2014;14(10):958-66.

11. Johnson NW, Gupta B, Speicher D, et al. Etiology and risk factors. In: Shah J, Johnson NW, editors. Oral and oropharyngeal cancer. Second ed. Boca Raton, FL: CRC Press; 2019. p. 19-94.

12. Hashibe M, Brennan P, Chuang SC, et al. Interaction between tobacco and alcohol use and the risk of head and neck cancer: pooled analysis in the International Head and Neck Cancer Epidemiology Consortium. Cancer Epidemiol. Biomarkers Prev. 2009;18(2):541-50.

13. Australian Institute of Health and Welfare. Alcohol, tobacco \& other drugs in Australia [Internet]. Canberra: Australian Institute of Health and Welfare, 2021. Available from: https://www.aihw.gov.au/reports/alcohol/alcohol-tobacco-other-drugs-australia (accessed Jan 2022)

14. National Health and Medical Research Council. Australian Guidelines to Reduce Health Risks from Drinking Alcohol. Commonwealth of Australia, Canberra: National Health and Medical Research Council, Australian Research Council and Universities Australia; 2020.

15. Haeggblom L, Ramqvist $T$, Tommasino $M$, et al. Time to change perspectives on HPV in oropharyngeal cancer. A systematic review of HPV prevalence per oropharyngeal sub-site the last 3 years. Papillomavirus Res. 2017;4:1-11.

16. Hocking JS, Stein A, Conway EL, et al. Head and neck cancer in Australia between 1982 and 2005 show increasing incidence of potentially HPV-associated oropharyngeal cancers. Br. J. Cancer. 2011;104(5):886-91.

17. Pytynia KB, Dahlstrom KR, Sturgis EM. Epidemiology of HPV-associated oropharyngeal cancer. Oral oncology. 2014;50(5):380-6.

18. Drake VE, Fakhry C, Windon MJ, et al. Timing, number, and type of sexual partners associated with risk of oropharyngeal cancer. Cancer. 2021;127(7):1029-38.

19. Gillison ML, D'Souza G, Westra W, et al. Distinct Risk Factor Profiles for Human Papillomavirus Type 16-Positive and Human Papillomavirus Type 16-Negative Head and Neck Cancers. JNCl. 2008;100(6):407-20.

20. Rissel CE, Richters J, Grulich AE, et al. Sex in Australia: first experiences of vaginal intercourse and oral sex among a representative sample of adults. Aust N Z J Public Health. 2003;27(2):131-7.

21. Rissel C, Heywood W, De Visser RO, et al. First vaginal intercourse and oral sex among a representative sample of Australian adults: The Second Australian Study of Health and Relationships. Sexual health. 2014;11(5):406-15.

22. Nielsen KJ, Jakobsen KK, Jensen JS, et al. The Effect of Prophylactic HPV Vaccines on Oral and Oropharyngeal HPV Infection-A Systematic Review. Viruses. 2021;13(7):1339.

23. Zhang Y, Fakhry C, D'Souza G. Projected Association of Human Papillomavirus Vaccination With Oropharynx Cancer Incidence in the US, 2020-2045. JAMA Oncology. 2021;7(10):e212907. 
24. Hall MT, Simms KT, Lew J-B, et al. Projected future impact of HPV vaccination and primary HPV screening on cervical cancer rates from 2017-2035: Example from Australia. PLoS One. 2018;13(2):e0185332.

25. Falcaro $M$, Castañon A, Ndlela $B$, et al. The effects of the national HPV vaccination programme in England, UK, on cervical cancer and grade 3 cervical intraepithelial neoplasia incidence: a registerbased observational study. The Lancet (British edition). 2021;398(10316):2084-92.

\section{Figures}

\section{Figure 1}

Joinpoint analysis of age-standardised incidence rates (ASR) per 100,000 population of OPC in Australian males from 1982-2017.

\section{Figure 2}

Joinpoint analysis of age-standardised incidence rates (ASR) per 100,000 population of OPC in Australian females from 1982 to 2017.

\section{Figure 3}

Joinpoint analysis of age-standardised incidence rates (ASR) per 100,000 population of OPC in Australian total from 1982 to 2017.

\section{Figure 4}

Age distributions for OPC cases in Australia, in 7-year periods, from 1983 to 2017 
Figure 5

Number of incident cases in each anatomical subsite of OPC in Australia, from 1982 to 2017. 